\title{
OBSERVATIONS ON THE WAVE AND SPIKE COMPLEX IN THE ELECTRO-ENCEPHALOGRAM
}

\author{
BY \\ ERIC C. O. JEWESBURY and M. J. PARSONAGE \\ From the Department of Applied Electro-shysiology, The National Hospital, Queen Square, London
}

The classical 3-per-second waves associated with negative spikes in the electro-encephalogram (EEG) have been well-recognized findings in cases of petit mal since they were first clearly described by Gibbs, Davis, and Lennox (1935) and again by Gibbs, Gibbs, and Lennox (1937). These latter describe the wave and spike pattern in 82 per cent. of cases where there is a history of petit mal alone and in 65 per cent. of cases where there is a history of grand mal in addition. In 6 per cent. of cases of grand mal, where clinical attacks of petit mal have not been perceptible, the wave and spike pattern is also found (Gibbs, Gibbs, and Lennox, 1943). These workers found the wave and spike phenomenon in only 1 in 500 cases of 7,600 non-epileptic neurological patients and normal controls, while Williams (1944) states that the only case in 8,000 in which he observed a wave and spike outburst in a non-epileptic subject was in the identical twin of an epileptic. The association of this EEG pattern with idiopathic epilepsy, particularly the petit mal variety, therefore needs no further emphasis.

There are some general aspects of the wave and spike complexes which have received little attention, and in the present paper an attempt has been made to describe some of these features by analysing a series of a hundred records which were selected purely on the grounds that they contained outbursts of typical wave and spike formations, the clinical aspect being largely a secondary consideration. Particular attention has been paid to the general character of the records, the incidence of the complexes, and their mode of development, excitation, duration, and relationship to clinical attacks.

\section{Method and Material}

Bipolar recordings of at least fifteen to twenty minutes' duration were obtained from a 3- or 6-channel inkwriting electro-encephalograph, two rows of saline pad electrodes being placed over each hemisphere. The subjects had taken no food for three hours beforehand. They lay comfortably with eyes closed during the recordings, which were made in four positions : two antero-posterior (parasagittal and temporal) and two transverse (frontal and central, central and post-central). The effect of opening and closing the eyes was noted in all cases, and overbreathing, as suggested by Lennox, Gibbs, and Gibbs (1936), was done for two and a half to three minutes by the majority of subjects.

One hundred consecutive records showing typical wave and spike formations were examined systematically. Atypical and abortive forms were disregarded. The subjects' were all known or suspect epileptics and there was an approximately even distribution between the sexes. The ages in seventy-five out of the hundred cases ranged from 5 to 20 years inclusive. Records were not usually made from children under the age of 5 years, and there were only three such in the present series. Eight of the patients were over 30 years old, the two eldest being aged 46 years.

Most of the subjects had been diagnosed clinically as suffering from simple petit mal, though some of these were also having occasional myoclonic or grand mal attacks. A small number of the subjects suffered either from grand mal alone, or attacks the nature of which was clinically somewhat doubtful.

\section{General Characters of the Records}

Before examining the wave and spike complexes themselves, the general characters of the records were first studied and compared.

In eighty-eight cases the dominant frequency in the post-central areas showed the characters and distribution of normal alpha rhythm. In fifty-seven of these, that is in just over half of the whole series, this rhythm was a prominent feature and had an amplitude of about 50 microvolts or more, whereas in only twelve cases was it irregular, ill-defined, or absent.

Apart from the wave and spike complexes, fiftyeight records were otherwise normal. Abnormal rhythms observed in the remaining forty-two cases were classified as follows : 
Delta range (1-4 c/sec), often seen as 3 $\mathrm{c} / \mathrm{sec}$ waves in both occipital regions Theta range (4-7 c/sec) Generalized dysrhythmia High voltage fast activity $\quad$. $\quad$. . 6 cases

Although 3-c/sec waves were noted, isolated kes were not seen in any of the records.

\section{Special Characters of the Wave and Spike Complexes}

Effect of Overbreathing.-In eighty cases typical wave and spike complexes occurred in the ordinary resting records, irrespective of whether they were produced by overbreathing. In the remaining twenty cases the wave and spike complexes were seen onily as a consequence of overbreathing. Overbreathing was done by eighty-two of the hundred patients, and in no less than seventy-four of these the complexes developed apparently as a result of this procedure. They appeared either during the course of overbreathing or very shortly afterwards, the former being much the commoner. In thirty-three cases wave and spike complexes appeared during both these periods, The importance of overbreathing in precipitating wave and spike outbursts is thus very evident.

Effects of Opening and Closing the Eyes.-The effect of voluntary opening and closing the eyes several times during the recording was noted in all cases. Wave and spike complexes occasionally developed within 5 seconds or less of these movements. In eight cases they followed opening, and in fifteen cases they followed closing of the eyes. This response might be seen once or several times in a single record. In four cases wave and spike complexes on occasion followed both opening and closing of the eyes. In three cases opening of the eyes inhibited an existing wave and spike outburst.

Incidence of Outbursts. - In the standard length of record before overbreathing the number of outbursts of wave and spike cumplexes varied considerably from case to case. The smallest number was one and the greatest twenty-nine. In ten records there were ten or more, but the average number of spontaneous outbursts in a single record was between three and four.

Duration of Outbursts.-The duration of wave and spike outbursts was also very variable. The shortest period was less than 1 second, but the longest was almost continuous and consisted of a series of long outbursts (often of half to one minute each). In the latter case each new outburst would appear either as the preceding outburst died away or just after it had done so. The average-duration of single outbursts per record was noted, and in only twenty cases did this amount to 15 seconds more. The average for the whole series 'was seconds.

The length of individual outbursts in a single record showed much variation, but in some cases there was a striking constancy. This applied both to the spontaneous outbursts and to those induced by overbreathing. A few examples will suffice. Thus, in one instance there were four spontaneous outbursts and two associated with overbreathing; the duration of each of them being 3 seconds. I $\bar{b}$. another record there were twenty-nine spontaneous outbursts-all brief, lasting between 1 and 3 second each. In another instance all nine spontaneou $\bar{s}$. outbursts and all six associated with overbreathin lasted approximately $2 \frac{1}{2}$ seconds. In another the duration of three spontaneous outbursts was 8 seconds, 6 seconds, and 5 seconds, and that of tw $\overrightarrow{0}$ outbursts with overbreathing 5 seconds each. In another record there were only two outbursts, eacte of 9 seconds, and in another only two outbursts: each of 15 seconds. In two other instances the duration of bursts was respectively 14 seconds; 14 seconds, 13 seconds, and 13 seconds, 13 secongs 12 seconds. It thus appeared that in some cassess there was a strong tendency for the length of the outbursts to be stabilized, and this applied whetef the duration of each outburst was long or shore -

Pattern of Outbursts.-In ninety-four cases $81 \mathrm{k}$ waves and spikes were entirely generalized, appea ing in all leads. In three cases the wave and siks pattern appeared in the frontal leads only. Fit three other cases the discharge was seen to be both generalized and localized at different times in the same record. The localized outbursts in these cases were frontal in one instance, occipital i another, and both frontal and occipital on differer occasions in the third.

In most cases the wave and spike outburs $\overrightarrow{\vec{E}}$ appeared abruptly in the record although they were sometimes heralded by a short run of irregula slow waves. Usually this irregularity of rhythm began and ended synchronously in all leads, but there was a tendency for the wave and spike conf plex in such cases to assume its characteristic forri more quickly and more clearly in the fronto. parietal leads. The wave and spike complexes also tended to persist longer in these leads, bu the generalized dysrhythmia usually ended as synchronously as it began. In about 10 per cents of cases the dysrhythmia was first and last manife in the fronto-parietal regions, and in 5 per cento. or less it was first and last seen in the occipitar leads. In the course of the longer bursts it wals noticeable, as pointed out by Jasper (1941), that 


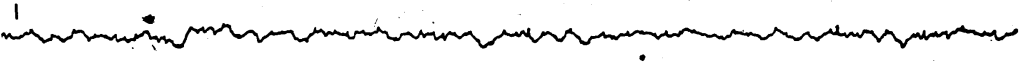

2

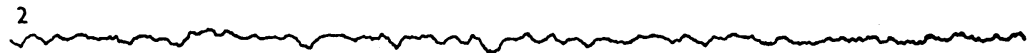

3

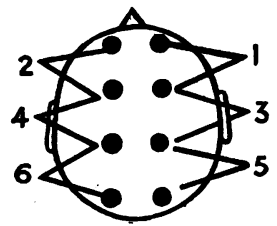

4
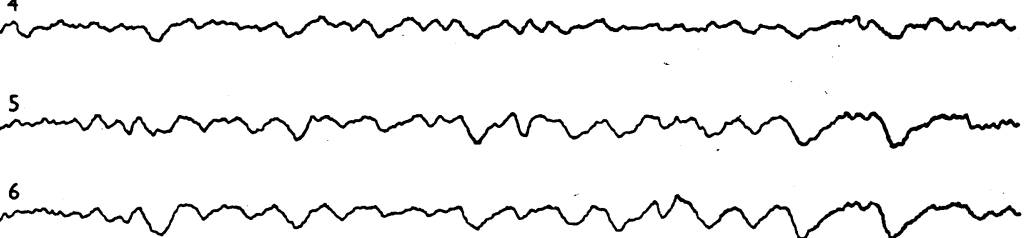

Seconds

$I=50 \mu V$.

FIG. 1.-3-4 c/sec activity in the occipital regions. A single outburst of wave and spike activity was seen in this record only during overbreathing.

the wave and spike complex tended to decrease slightly in frequency, for example, from between 3 and $4 \mathrm{c} / \mathrm{sec}$ at the onset to between 2 and $3 \mathrm{c} / \mathrm{sec}$ at the end of a paroxysm. The spike component of the complex frequently appeared to become "locked" in a fixed position relative to the slow wave and both frequencies diminished together. Other small spikes or sharp waves were sometimes seen at varying points on the slow wave during the burst, and the spikes sometimes disappeared before the slow waves. The pattern of wave and spike was least characteristic in the occipital leads.

\section{Relationship of Complexes to Clinical Attacks}

Twenty-nine patients showed obvious clinical attacks of petit mal, associated always with wave and spike complexes during the recording. It was noticeable that almost all of the EEG outbursts associated with a clinical attack were considerably longer than the average duration of 8 seconds. To this there were only two clear exceptions. First, clinical petit mal sometimes occurred in association with brief, irregular, repeated bursts of wave and spike patterns during the course of overbreathing. Secondly, clinical attacks were associated in one case with brief, repetitive, spontaneous outbursts of constant duration.

In this case there were sixteen spontaneous bursts and three bursts associated with overbreathing, each of them lasting 4 to 5 seconds and each accompanied by clinical petit mal. There were no bursts of longer duration in this case, and the tendency to a fixed pattern of discharge, accompanied by a clinical attack, seemed strong. This patient was having petit mal attacks almost every day. On the other hand, fairly long outbursts of wave and spike complexes sometimes occurred without any evidence of a clinical attack being detected during the routine recording. For example, in one instance an outburst lasted over 30 seconds without any evidence of a clinical attack. No special methods were used for the detection of an attack, however, so that probably the mildest changes in consciousness were sometimes overlooked.

It was observed that, as a rule, there was little or no relationship between the frequency of petit mal attacks, as given in the patient's history, and the number of wave and spike outbursts occurring in the EEG. The liability to attacks seemed to be rather more closely related to the duration than to the number of outbursts which were found in the record. Thus, one long burst of waves and spikes was found to be more usually associated with a history of frequent clinical attacks as well as with an attack during the recording than were several short outbursts. In this series, however, no constant relationship was found between the severity of the clinical symptoms and the extent of the EEG changes.

In the present investigation we have not found any focal or other distinctive features in the records of patients having extremely frequent and brief petit mal attacks, clinically compatible with the diagnosis of pyknolepsy. Grey Walter (1938) has referred to a delta focus situated in the occipital poles in cases of so-called pyknolepsy of children 
and in the present series there were eight patients whose records showed delta activity localized to the occipital regions (see Fig. 1). Clinically, however, this group showed no special features which in any way distinguished them from the remainder of the patients.

There were two patients in the series whose records showed localized wave and spike outbursts in the occipital regions. The first was a boy aged 9 years, who had been having petit mal attacks ("sometimes 7 or 8 while having dinner"), for two years; these had first begun two months after mild concussion, but there were no abnormal clinical or radiological findings. It was of interest that his mother had suffered from fainting attacks when prégnant. The other patient was aged 10 years, had suffered from ordinary petit mal attacks for three years, and at the time of recording was having about one attack daily. These case histories were entirely comparable with those whose records showed no sort of occipital focus. Similarly, there were no special clinical features among the five cases whose records appeared to show a focus of wave and spike activity in the frontal regions.

In four cases of petit mal the EEG was recorded both before and during the administration of "tridione." In each instance wave and spike outbursts were found before treatment was begun. Three of the cases showed a satisfactory clinical response to the administration of the drug in the usual dosage of one capsule $(0.3 \mathrm{~g}$.) given thrice daily. In the first case wave and spike outbursts disappeared from the record, but in the second they persisted unchanged, while in the third they not only persisted but were of even longer duration than originally. The fourth case showed no clinical improvement with " tridione," although the EEG showed increased stability and at least temporary abolition of wave and spike outbursts.

\section{Discussion}

Several interesting points emerge from a study of these records, and are considered worthy of further discussion. Moreover, from the data it is possible to construct a composite picture of an "average" type of record containing wave and spike activity likely to be met with in a case of already diagnosed or suspect petit mal.

- As regards the general characters of the records, it is to be noted that in rather more than half the cases these were quite normal in appearance, apart from the presence of wave and spike activity. In nearly 90 per cent. of cases there was a wellmarked normal alpha rhythm which was dominant in the post-central areas, and in 57 per cent. of cases had an amplitude of 50 microvolts or more.
Of the additional abnormalities observed, commonest (seventeen out of forty-two cases) wos the presence of delta activity usually occurring 5 as bilaterally synchronous medium voltage wavescat about 3 c/sec in the occipital regions. The nest commonest abnormality was the presence of theta activity considered excessive in amount for the gige in question (thirteen out of forty-two cases). Lastly, it may be emphasized that the occurrefice of high voltage fast activity was a comparative rarity (6 per cent. of cases), while a generali $\stackrel{\text { fd }}{\text { d }}$ dysrhythmia containing several differing frequençes was seen in only 9 per cent. of the cases.

Overbreathing was frequently associated with appearance of wave and spike outbursts, and The fact that in twenty cases these appeared only when the patients were made to carry out this procedure is ample testimony to its value in the confirmation of a diagnosis which may be otherwise doubtfitl. There is sometimes great difficulty in persuadigng small children to overbreathe satisfactorily, fut every effort should be made to obtain their gooperation, since this procedure may yield information which cannot readily be obtained by otther means.

It was notable that in nineteen of the huoded cases wave and spike outbursts seemed to precipitated by either opening or closing theceyes, more commonly the latter (see Fig. 2): Any form of attention, visual or otherwise, is well knognoto abolish normal alpha rhythm, and it may bet altered visual attention or mental concentrition was a factor in these cases. Since, however, Inese outbursts of wave and spike complexes were often quite transient and might be related either to opening or to closing of the eyes, it is possifle that they were precipitated by other factors associated with the voluntary movement of the eyes or lids rather than by simple visual attention.

The figures show that there is no constant relationship between the length and number of the wave and spike outbursts in the EEG and 垌e occurrence of a clinical attack during the recordifing. It is also clear that from the study of a single record one cannot estimate accurately what may fbe happening clinically in terms of the number:of daily, weekly, or monthly attacks. In further support of this point, we may quote the case a youth aged 21, not included in this series, wo had had typical petit mal attacks regularly since the age of 10 years, and who at the time of examination was having two to five attacks daily. Fis EEG showed a very. mild generalized abnormatity of non-specific type but nothing suggestive of wive and spike activity.

These observations are in keeping with 密e 


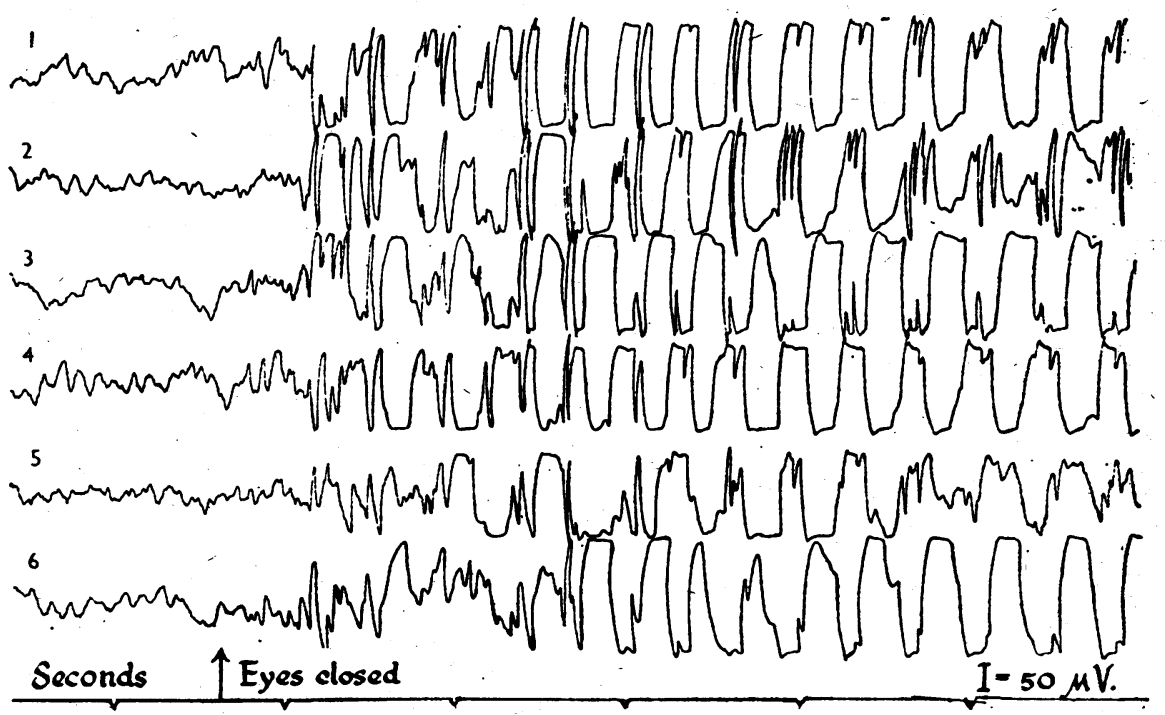

Fig. 2.-Development of wave and spike outburst immediately after closing the eyes. In this case voluntary closure of the eyes was followed on nine out of sixteen occasions by well-marked wave and spike complexes. On four of these occasions a clinical petit mal attack was observed.

suggestion of Williams (1949) that there are at least two main factors which interact in the production of clinical attacks ; on the one hand there is the dysrhythmia in the EEG, and on the other hand there may be an additional hidden factor of " convulsibility," representing a liability to respond to appropriate dysrhythmia with the production of major or minor fits, as the case may be.

The fact that twenty-nine patients are noted as having had obvious clinical attacks of the petit mal variety during the recording is one which requires considerable qualification, because the clinical manifestations of these attacks are often so slight that they can easily pass unobserved when the attention of the operator is temporarily distracted from the patient, as indeed must often be the case. Fortunately this difficulty is rarely of any practical importance.

The results of the present investigation lend no support to the conception of pyknolepsy as a condition entirely independent from petit mal, since the records of the patients who had been diagnosed as suffering from the former condition showed no focal or other special featurès to distinguish them from the remainder. The term " pyknolepsy" (meaning crowded or condensed seizures) was first introduced by Sauer. (1912). The attacks to which he applied it had been previously described by numerous writers, particularly by Fürstner (1896), who regarded them as probably hysterical in origin, and by Friedmann (1906) who called them " non-epileptic absences or short narcoleptic attacks." The first English account of pyknolepsy was given by Adie (1924) who recommended the use of the name because it was purely descriptive and non-committal. He questioned the then prevalent view that pyknolepsy was a disease sui generis, and subsequent opinion has more and more come to regard the condition as a form of idiopathic epilepsy. Jelliffe and Notkin (1935), in a review of the literature, concluded that most of the reasons offered by the various workers for regarding pyknolepsy as a separate entity could hardlý bear criticism. More recently Owen and Berlinrood (1942) made a clinical and electroencephalographic study of eight cases of pyknolepsy. They found typical wave and spike patterns of the petit mal variety in the records of five cases, and they also noted that attacks could be precipitated by overbreathing; their conclusion was that pyknolepsy was no more than a form of petit mal epilepsy. We are in agreement with this view and there seems little to commend the retention of the term pyknolepsy as distinct from petit mal.

The number of cases treated with "tridione" in the series is too small to be of much value, except perhaps to emphasize the fact that the appearances in the EEG do not necessarily run parallel with the clinical response. They illustrate the exceptions to the general rule observed by Lennox (1947) that disappearance of clinical petit mal is usually followed by reduction or disappearance of wave and spike 

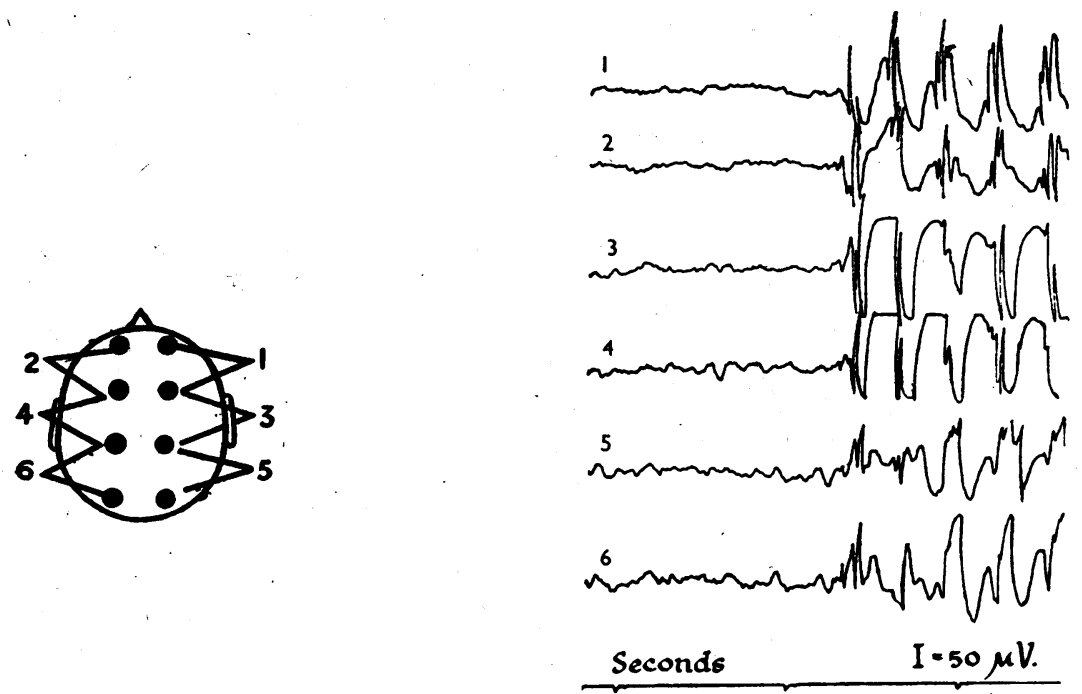

Fig. 3.-Spontaneous wave and spike outburst, showing characteristic abrupt onset.

and spike outbursts with occur in the resting record, and in a stọl greater proportion the will also develop as 9 result of overbreathing. There will be almost one in five chance off their being precipitated by opening or closing the eyes. In the restiogs record there will protably be three or fow outbursts, each lastififs rather - less than te seconds, and not often more than fifteen. Typically each wave and spike outburst begiass fairly abruptly as a viplaterally synchronouss rhythm of high voltage,

activity in the EEG. Moreover, the observations in this small series suggest that the findings in the EEG alone cannot be taken as a reliable indicator of a favourable response to "tridione" therapy, and we' cannot therefore agree with Peterman (1948) that in idiopathic epilepsy the EEG " provides the only objective evidence for a true evaluation of treatment."

Finally, taking the data as a whole, it is interesting to construct a hypothetical " average" type " of wave and spike record. In the first place it is rather more likely than not to be an otherwise normal record. In eight out of ten cases the wave in which the wayes and spikes are "locked" together as recurring complexes (Fig. 3). Thoise complexes usually assume their most charactenistic form in the fronto-parietal regions. Their offequency in the initial stages is at $3.4 \mathrm{c} / \mathrm{sec}$, बెbut gradually slows down in the later stages to 3 and sometimes even $2 \mathrm{c} / \mathrm{sec}$. Finally, the spikes begrim to disappear and the outburst dies away in the form of a few slow waves of decreasing amplitude, ending synchronously in all areas (Fig. 4). Duringo trie recording there is perhaps about a one in three chance of there being easily detectable signs of a clinical petit mal attack.
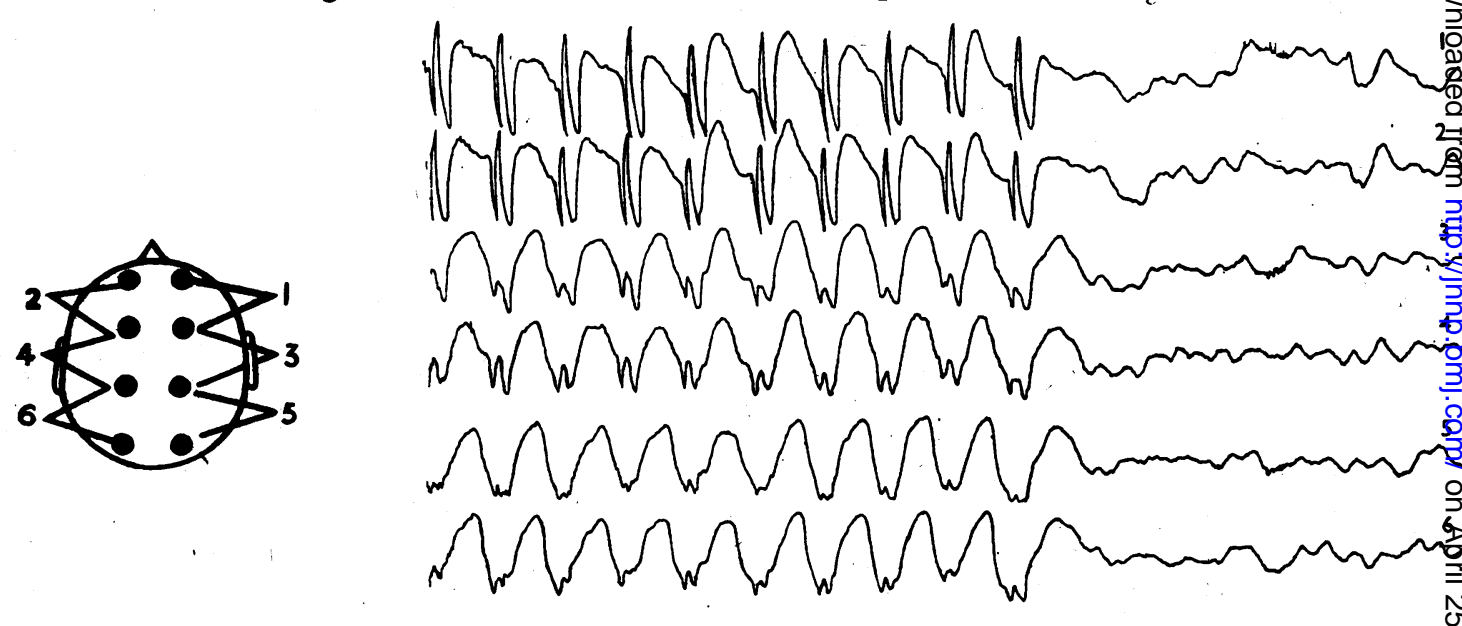

Seconds

$I=50 \mu N$

FIG. 4.-Characteristic ending of wave and spike outburst. 


\section{Summary and Conclusions}

The EEGs of one hundred cases showing typical wave and spike complexes were examined. Seventyfive of the patients were aged from 5 to 20 years inclusive.

Normal alpha rhythm was seen in eighty-eight cases. Apart from the wave and spike complexes fifty-eight records were otherwise normal. Abnormal rhythms seen are described.

In eighty cases the wave and spike complexes occurred without overbreathing, but in twenty cases they appeared only when the patient overbreathed.

Overbreathing was done by eighty-two of the patients, and seventy-four of these developed wave and spike complexes in relation to the overbreathing.

The effect of opening and closing the eyes was observed in all the hundred cases. In eight cases wave and spike complexes followed opening, and in fifteen they followed closing of the eyes. In four of these cases there was a response both to opening and closing movements. In three cases opening of the eyes was seen to inhibit an existing wave and spike outburst.

The average number of wave and spike outbursts in a record before overbreathing was between three and four.

The wave and spike outburst might last for less than a second or be almost continuous for several minutes. The total average duration of a single outburst per record was 8 seconds. The length of individual outbursts in a single record showed much variation but in some cases there was a striking constancy of duration of the outbursts.

In ninety-four cases the wave and spike complexes were entirely generalized. In three cases localized as well as generalized outbursts were seen. In three cases the complexes were localized to the frontal regions only. No special clinical features distinguished the few cases in which localized outbursts occurred.

In most cases the wave and spike outbursts appeared abruptly and synchronously in all leads. The complexes themselves might be preceded and followed by a short burst of irregular waves, and there was then a tendency for the wave and spike complex to assume its characteristic form more rapidly and more clearly in the fronto-parietal regions. The wave and spike complexes tended to persist longest in the area in which they were first seen. In about 10 per cent. of cases the outburst was predominantly fronto-parietal, and in 5 per cent. or less it was predominantly occipital.
During long outbursts the wave and spike complex tended to decrease slightly in frequency.

Twenty-nine patients showed obvious clinical attacks of petit mal during the recording. All clinical attacks were associated with wave and spike outbursts, and these were usually of longer duration than the average. The liability to clinical attacks seemed to be rather more closely related to the duration than to the number of outbursts in the record, but there was no constant relationship between the severity of the clinical symptoms and the extent of the EEG changes.

So-called "pyknolepsy" could not be sharply distinguished from petit mal by the EEG any more than it could by clinical standards.

The clinical response of cases of petit mal to treatment with "tridione" was not necessarily associated with parallel changes in the EEG.

A random twenty minutes EEG - record is of limited value in assessing the frequency of attacks, prognosis, or progress of a patient with petit mal. It may, however, have great-diagnostic value in doubtful cases.

We wish to thank Dr. Denis Williams and Dr. W. A. Cobb for their helpful criticism in the preparation of this paper.

\section{REFERENCES}

Adie, W. J. (1924). Brain, 47, 96.

Friedmann, M. (1906). Dtsch.Z. Nervenheilk., 30, 462. Fürstner. (1896). Arch. Psychiat., 28, 494.

Gibbs, F. A., Davis, H., and Lennox, W. G. (1935). Arch. Neurol. Psychiat., Chicago, 34, 1133.

, Gibbs, E. L., and Lennox, W. G. (1937). Brain, 60, 377.

$$
\text { Chicago, 50, } 111 .
$$

(1943). Arch. Neurol. Psychiat.,

Jasper, H. H. (1941). In "Epilepsy and Cerebral Localization" by Penfield, W., and Erickson, T. C. London. Chapter 14.

Jelliffe, S. E., and Notkin, J. (1935). Arch. Neurol. Psychiat., Chicago, 33, 752.

Lennox, W. G. (1947). J. Amvr. med. Ass., 134, 138. -, Gibbs, F. A., and Gibbs, E. L. (1936). Arch. Neurol. Psychiat., Chicago, 36, 1236.

Owen, J. W., and Berlinrood, L. (1942). Amer. J. Psych., 98, 757.

Peterman, M. G. (1948). J. Amer. med. Ass., 138, 1012. Sauer, H. (1912). Mschr. Psychiat. Neurol., 40, 27.

Walter, W.-G. (1938). J. Neurol. Psychiat., n.s., 1, 359. Williams, D. (1944). Brain, 67, 10.

(1949). Unpublished observations at meeting of International League Against Epilepsy (British Branch). Jan. 7, 1949. 